\title{
Consensos e dissensos na indicação e continuidade da terapia nutricional enteral nos cuidados paliativos de pacientes com doenças crônicas não transmissíveis
}

Agreements and disagreements on indication and continuity of enteral nutritional therapy in palliative care patients with non-communicable diseases

Juliana Maura Ferreira de Castro ${ }^{1}$, Vera Silvia Frangella ${ }^{1}$, Marjorie Terumy Hamada²

${ }^{1}$ Centro Universitário São Camilo (CUSC) - São Paulo (SP), Brasil.

${ }^{2}$ Hospital Israelita Albert Einstein (HIAE) - São Paulo (SP), Brasil.

DOI: http://dx.doi.org/10.7322/abcshs.v42i1.951

\section{RESUMO}

As doenças crônicas não transmissíveis (DCNT) representam as principais causas de internações hospitalares, geram impactos socioeconômicos e comprometimento da qualidade de vida em virtude de suas sequelas e incapacidades, justificando a importância e necessidade crescente de associação dos cuidados paliativos ao tratamento curativo. Esta revisão narrativa visa apresentar os consensos e dissensos sobre a indicação e a continuidade da terapia nutricional enteral (TNE) nos cuidados paliativos de pacientes com DCNT. Embasou-se em publicações oficiais sobre o tema e 15 artigos divulgados nas bases de dados: LILACS, SciELO, PubMed, MEDLINE, Cochrane Library e Science Direct, entre os anos de 2005 e 2016. Os objetivos do suporte nutricional nos cuidados paliativos desses pacientes variam com a evolução da doença. O suplemento nutricional oral é indicado para complementar a ingestão alimentar oral insuficiente, reduzindo custos hospitalares e favorecendo melhoras clínicas e funcionais. $\mathrm{Na}$ ingestão alimentar menor do que $60 \%$ e sem previsão de evolução, é indicada a TNE dentro dos três primeiros dias, mas sua continuidade em doenças avançadas permanece controversa. $\mathrm{Na}$ fase terminal, prioriza-se o conforto, o alívio dos sintomas e não mais a adequação nutricional. Nessa fase, a nutrição e hidratação artificiais podem não ser benéficas. Dessa forma, a nutrição nos cuidados paliativos é individualizada, depende do estágio da doença e visa promover a qualidade de vida. As tomadas de decisão devem envolver a vontade do paciente e de seus familiares, considerando os princípios de autonomia, beneficência, não maleficência e justiça.

Palavras-chave: cuidados paliativos; doença crônica; terapia nutricional.

\begin{abstract}
Non-communicable diseases (NCD) are the leading cause of hospital admissions, result in socioeconomic impacts and prejudice on quality of life due to after-effects and disabilities, justifying the importance and the increasing need to associate palliative care with curative treatment. This narrative review aims to introduce agreements and disagreements on indication and continuity concerning enteral nutritional therapy (ENT) in palliative care patients with NCD. This paper was based on official publications related to the topic and 15 articles found on LILACS, SciELO, PubMed, MEDLINE, Cochrane Library and Science Direct databases, between the years 2005 and 2016. In these patients, the objective of nutritional support in palliative care varies according to the evolution of the disease. Oral nutritional supplement is indicated to supplement insufficient oral food intake, reducing hospital costs and favoring clinical and functional improvements. When food intake is less than $60 \%$ and without evolution prospects, ENT is indicated within the first 3 days, but withholding and withdrawing it in advanced diseases remains controversial. In terminal phase, comfort and relief of symptoms are the priority instead of nutritional adequacy. On this stage, artificial nutrition and hydration may not be beneficial. Therefore, nutrition in palliative care is individualized, depends on the stage of the disease and aims to promote life quality. Decisions must be taken considering patient and relatives' will, principles of autonomy, beneficence, no maleficence and justice.
\end{abstract}

Keywords: palliative care; chronic disease; nutrition therapy. 


\section{INTRODUÇÃO}

Atualmente, as doenças crônicas não transmissíveis (DCNT) representam a maior causa de morbimortalidade mundial e de perda da qualidade de vida em virtude de sequelas e incapacidades, sendo responsáveis por intensos impactos socioeconômicos. No Brasil, em 2011, foram registrados aproximadamente 1,1 milhão de mortes com destaque para as doenças cardiovasculares $(30,4 \%)$, neoplasias $(16,4 \%)$, doenças respiratórias $(6,0 \%)$ e diabetes mellitus $(5,3 \%)^{1}$.

Em síntese, as DCNT atingem todos os indivíduos de qualquer classe socioeconômica, principalmente grupos vulneráveis como os idosos e a população de baixa escolaridade e renda. Essas doenças estão entre as maiores causas de internações hospitalares e são consideradas um problema para todos os sistemas de saúde públi$\mathrm{ca}^{1,2}$. Mediante o exposto, é necessária a associação da abordagem paliativa ao tratamento curativo, o qual perde a capacidade de oferecer controle razoável à medida que a doença progride, tornando os cuidados paliativos uma necessidade crescente e absoluta ${ }^{3}$.

Historicamente, os cuidados paliativos eram oferecidos principalmente aos pacientes com câncer. Entretanto, vários indivíduos vivem por um longo período com outras doenças limitativas e atualmente esses cuidados vêm sendo considerados instrumento de apoio para todos aqueles com doenças crônicas evolutivas e progressivas, com prognóstico de meses ou anos ${ }^{4,5}$.

Os cuidados paliativos consistem na assistência integral promovida por uma equipe multidisciplinar, com o objetivo de proporcionar melhoria na qualidade de vida dos pacientes e seus familiares diante de doenças que ameaçam a continuidade da vida, por meio da prevenção, alívio do sofrimento, avaliação impecável, tratamento da dor e de outros problemas de natureza física, psicossocial e espiritual. Além disso, a abordagem paliativa deve ser introduzida em todos os estágios da doença, desde a descoberta do diagnóstico e tratamento, em sua progressão e no término da vida ${ }^{6}$.

Inquestionavelmente, todo ser humano necessita da nutrição e hidratação para sobreviver. Enquanto cada indivíduo possui a capacidade de se hidratar adequadamente, consumir alimentos para cobrir as necessidades nutricionais e deseja fazê-lo, não há necessidade de intervenção. Contudo, os problemas surgem quando isto não é possível ou a ingestão de nutrientes e líquidos é insuficiente. Dessa forma, cabe aos profissionais de saúde fornecer o tratamento adequado, incluindo a terapia de nutrição e hidratação ${ }^{7}$.

O paciente em cuidados paliativos comumente apresenta inapetência alimentar, perda ponderal e queixas decorrentes dos eventos adversos do tratamento, como náuseas e vômitos, diarreia, obstipação, xerostomia, entre outros fatores que interferem no estado nutricional ${ }^{8}$. Diante desse enfoque, a nutrição é considerada importante por auxiliar na ação preventiva, possibilitando meios e vias de alimentação, contribuindo para o retardo de comprometimentos nutricionais como a síndrome de anorexia-caquexia e reduzindo os eventos adversos do tratamento 9 .
Os objetivos do suporte nutricional nessa modalidade terapêutica irão variar de acordo com a evolução da doença. Quando o paciente é diagnosticado e está em tratamento, a conduta é baseada na oferta de nutrientes em qualidade e quantidade adequadas, visando restaurar ou manter o estado nutricional, reparar tecidos, prevenir infecções e promover uma sensação de bem-estar. Entretanto, à medida que o fim se aproxima, o alimento continua a ser ofertado, porém o objetivo principal está direcionado à qualidade de vida, ao conforto e ao alívio do sofrimento, não mais como um tratamento ativo para promover a adequação nutricional ${ }^{5}$.

A administração da dieta em cuidados paliativos pode ser realizada por via oral, sonda nasoentérica, gastrostomia e, menos frequente, por via parenteral ${ }^{5}$. Em relação à administração da terapia nutricional enteral (TNE) e/ou terapia nutricional parenteral (TNP), há ainda questões sobre sua introdução ou suspensão que geram muitas dúvidas, levando médicos e nutricionistas a questionarem seu real benefício ao paciente em cuidados paliativos, pois terapias nutricionais agressivas podem tornar o tratamento árduo e estressante ${ }^{8}$.

A indicação e a continuidade da TNE nos cuidados paliativos é um tema ainda considerado complexo e controverso, tornando-o relevante aos profissionais de saúde mediante sua importância na prática clínica. Perante o panorama descrito, este artigo visa apresentar os consensos e dissensos descritos em literatura científica sobre a indicação e a continuidade da TNE nos cuidados paliativos de pacientes com DCNT.

\section{MÉTODOS}

Esta revisão bibliográfica do tipo narrativa ${ }^{10}$ baseou-se no rastreamento de informações nas bases de dados, tais como: LILACS, SciELO, PubMed, MEDLINE, Cochrane Library e Science Direct. Para tanto, foram selecionados materiais divulgados entre os anos de 2005 e 2016, aplicando-se a lógica booleana com os conectores and e or e os seguintes descritores da saúde: cuidados paliativos or nutrição enteral, terminalidade and cuidados paliativos, doença crônica and assistência paliativa, dentre outros.

Para compor este estudo foram selecionados 15 artigos (7 nacionais e 8 internacionais), os quais foram lidos na íntegra para a construção de seus fichamentos e elaboração de planilha resumo em prol da análise e organização do conteúdo produzido.

Por fim, foram utilizados cadernos científicos elaborados pelo Ministério da Saúde, Conselho Regional de Medicina do Estado de São Paulo, Academia Nacional de Cuidados Paliativos e World Health Organization (WHO), bem como guidelines produzidos pela European Society for Parenteral and Enteral Nutrition (ESPEN), American Society for Parenteral and Enteral Nutrition

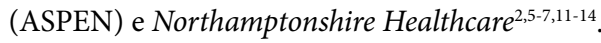




\section{Terapia nutricional enteral nos cuidados paliativos}

Os hábitos e as crenças alimentares são formados na infância e estão intimamente associados à família e à cultura ${ }^{4}$. Dessa forma, o consumo alimentar não pode ser considerado apenas como um meio de subsistência, visto que está vinculado a fatores afetivos que proporcionam experiências interpessoais de conforto, as quais não são alteradas quando um indivíduo é acometido por uma doença grave ${ }^{5}$.

Inevitavelmente, o paciente em cuidados paliativos possui sua jornada marcada por uma série de perdas gradativas, dentre elas as funções sensoriais e do sistema digestório ${ }^{5}$. Diante desse enfoque, o nutricionista auxiliará esses indivíduos balanceando de forma individualizada as recomendações dietoterápicas conforme os sintomas apresentados, proporcionando maior qualidade de vida ${ }^{8}$.

Em cuidados paliativos, há o dilema em relação ao emprego da dieta por via oral (VO), TNE e/ou TNP aos pacientes ${ }^{8}$. Na medida do possível, a VO sempre será preferencial, desde que o paciente esteja de acordo, com o trato gastrintestinal (TGI) íntegro e apresente condições clínicas para realizá-la ${ }^{11}$.

O uso do suplemento nutricional oral (SNO) é indicado quando a ingestão alimentar total pela VO é insuficiente, o paciente apresenta condições de ingestão por essa via e há integridade do TGI. Para quadros nos quais a ingestão alimentar é menor do que $60 \%$ e sem previsão de evolução, recomenda-se a TNE dentro dos três primeiros dias ${ }^{12}$.

A TNP é pouco aplicada em indivíduos com doença avançada. Pode ser indicada quando a utilização da TNE não for possível (quando não há funcionalidade do TGI) e/ou segura, desde que contribua para a qualidade de vida do paciente. Além disso, é necessário avaliar seus reais benefícios, visto que pode estar associada à atrofia intestinal, hiperglicemia, aumento do risco de complicações infecciosas e de mortalidade em pacientes com doenças graves ${ }^{11,13}$.

\section{Terapia nutricional enteral nos cuidados paliativos de doenças crônicas não transmissíveis: do diagnóstico ao tratamento}

O plano inicial de cuidados nutricionais para o paciente diagnosticado com uma doença crônica em que a morte não está iminente deve incluir triagem nutricional para determinar o risco de depleção, bem como providenciar estratégias para cada mudança futura. No estágio inicial da doença, encontrar e solucionar problemas nutricionais contribuem para a manutenção ou ganho ponderal do paciente, melhoram a resposta ao tratamento e reduzem complicações ${ }^{5,14}$.

Para minimizar a perda ponderal, promover a reparação e a regeneração de tecidos danificados é indispensável uma dieta rica em calorias e proteínas. Os pacientes que ingerem quantidades suficientes para manter seu senso de autonomia e qualidade de vida, por conseguinte, melhoram seu bem-estar. No entanto, muitos indivíduos que se alimentam de forma inadequada necessitam de suporte alimentar adicional e os SNO podem auxiliar neste processo ${ }^{4}$.

O gasto energético em pacientes com doença pulmonar obstrutiva crônica (DPOC) é elevado quando comparado a indivíduos saudáveis; e em virtude do esforço respiratório e das internações frequentes associadas a exacerbações agudas, pode ocorrer um balanço energético negativo. Diante desse enfoque, o suporte nutricional é considerado um aspecto importante nos cuidados paliativos desses pacientes, visto que a perda de peso e de massa muscular esquelética são fortes preditores de mortalidade na DPOC ${ }^{15}$.

$\mathrm{Na}$ Espanha foi realizado um estudo prospectivo, randomizado e multicêntrico por 12 semanas consecutivas, com 24 pacientes ambulatoriais portadores de DPOC estável e depleção nutricional. Foram avaliados os efeitos da ingestão alimentar complementada por SNO hipercalóricos e hiperproteicos na qualidade de vida desses pacientes e a eficácia da repleção nutricional sobre outras variáveis (peso corporal, força de preensão manual e limitação do fluxo de ar). Após 12 semanas de intervenção, foi observada melhora significativa na qualidade de vida desses pacientes, com o aumento da sensação de controle da doença pela redução dos sintomas de dispneia e fadiga, aumento do peso corporal, diminuição da limitação do fluxo de ar e melhora na força de preensão manual ${ }^{16}$.

Existem evidências crescentes sugerindo que o uso apropriado de SNO pode beneficiar o paciente favorecendo melhoras clínicas e funcionais, além de reduzir custos hospitalares ${ }^{17}$. Contudo, a administração de SNO deverá ser realizada sob a orientação de um nutricionista e com acompanhamento regular ${ }^{14}$.

\section{Terapia nutricional enteral nos cuidados paliativos de doenças crônicas não transmissíveis: da progressão à fase terminal}

A ideia de abstinência alimentar proporciona um sofrimento adicional para o paciente e seus familiares, visto que a progressão da doença ocasiona a perda de peso com impacto nas condições físicas, clínicas e psicossociais, as quais podem comprometer a qualidade de vida do indivíduo em cuidados paliativos ${ }^{9}$. Nessa condição, o objetivo da terapia nutricional deve priorizar o conforto e o alívio dos sintomas, não mais a adequação nutricional ${ }^{14}$.

A indicação e a continuidade da TNE em doenças avançadas ainda permanecem controversas ${ }^{11}$. Para pacientes com idade e demência avançada, a introdução e a continuidade da TNE por sonda ou gastrostomia, apesar de ser apropriada em alguns casos, priva o indivíduo da sensação de paladar, favorecendo a diminuição de sua dignidade e autoestima. Em geral, essa situação proporciona maior agitação, desconforto e necessidade de sedação nesses pacientes ${ }^{5,18}$.

Conforme a American Geriatrics Society ${ }^{19}$, em populações vulneráveis como os idosos demenciados em estágio avançado, os tubos de alimentação não são recomendados, pois sua inserção tem 
sido associada a reincidências de úlceras por pressão e ao aumento dos cuidados em saúde.

Foi realizado na Espanha um estudo prospectivo e observacional, com 67 pacientes idosos em demência avançada, $\geq 65$ anos e que faziam uso de dieta por VO e TNE por sonda nasogástrica. Foram analisados fatores de sobrevivência no período de 832 dias. Os resultados evidenciaram que a TNE por sonda nasogástrica não melhorou o estado nutricional e contribuiu para a redução da sobrevivência nesses pacientes (mediana de 799 dias e média de 676,8) devido ao aumento de complicações clínicas, dentre elas a pneumonia ${ }^{20}$.

Nos cuidados paliativos em pacientes crônicos, o objetivo final sempre será o paciente e seus familiares. Dessa forma, as condutas e o planejamento dos cuidados são concentrados nesses indivíduos e podem mudar com o passar do tempo. É inadequado, por exemplo, não indicar a TNE para um paciente demenciado, mesmo que os benefícios sejam incertos, pois faz parte da decisão para uma melhor conduta considerar as múltiplas circunstâncias em cada momento da evolução na doença de base, levando em consideração que diferentes enfermidades têm formas próprias de evolução esperadas 5 .

$\mathrm{Na}$ fase terminal da doença, o alimento possui uma importância simbólica na provisão da nutrição e hidratação do paciente. Nessa fase, alguns indivíduos sofrem com a alimentação por VO devido a desconfortos abdominais e náuseas, mas a mantêm apenas para agradar ou tranquilizar seus familiares ${ }^{9}$. Assim, os objetivos na fase terminal são minimizar o estresse e maximizar o conforto, sendo fundamental esclarecer aos familiares a importância de sua compreensão e a adequada participação na assistência a esses pacientes ${ }^{5}$.

A intervenção nutricional nessa fase deve ter como foco principal o prazer que os alimentos e bebidas proporcionam ao paciente e o alívio da pressão de manter uma dieta normal. Já os SNO podem ser benéficos para alguns indivíduos por motivos psicológicos, sendo necessário esclarecer que sua ingestão não irá melhorar o estado nutricional ${ }^{14}$.

A retenção ou suspensão do suporte nutricional de pacientes em fase terminal é um dilema ético que deve levar em consideração princípios de autonomia, beneficência, não maleficência e justiça. A beneficência se refere a não proporcionar sofrimento ao paciente, a não maleficência, a não o prejudicar, e a justiça visa fornecer no tempo restante de vida desse paciente bem-estar e conforto. Desse modo, para todos que recusarem a nutrição e hidratação artificiais (NHA) devem ser oferecidos cuidados paliativos com o objetivo principal de aliviar os sintomas físicos e psicológicos, incluindo suporte emocional e espiritual ${ }^{21}$.

A NHA pode não ser benéfica para pacientes em fase terminal, não aumentar a sobrevida ou o conforto do paciente, porém, muitas pessoas consideram mais aceitável a continuidade do tratamento do que sua suspensão, crendo que a decisão de retirada levaria o paciente à morte. Contudo, a suspensão da NHA não é o que vai originar a morte do paciente e sim a doença de base que atua como fator determinante ${ }^{21}$.
Foi realizado na Holanda um estudo prospectivo e observacional por 42 dias em 32 instituições de longa permanência, com 190 idosos em demência grave, no qual a NHA foi suspensa em 178 indivíduos e introduzida em 12 . Os resultados mostraram que eventos adversos como dispneia e agitação foram associados a elevados níveis de desconforto na população estudada, o qual diminuiu após a decisão da suspensão da $\mathrm{NHA}^{22}$.

Já para pacientes com câncer avançado, para os quais apenas a medicação paliativa é considerada, as evidências dos benefícios da NHA em prolongar a vida são menores ${ }^{5}$. Entretanto, sob acompanhamento e orientação individualizada, pacientes com câncer esofágico avançado, inoperável e sem perspectivas de retomada da ingestão oral, podem ser beneficiados com a introdução da gastrostomia endoscópica percutânea (PEG), levando em consideração que a maioria desses indivíduos sofre de disfagia significativa, perda ponderal e desnutrição, muitas vezes agravada pelo tratamento quimio e radioterápico ${ }^{23}$.

Um estudo realizado pelo Grupo de Estudo de Nutrição Entérica (GENE) avaliou a evolução clínica e nutricional de 15 pacientes adultos e idosos, com câncer de esôfago inoperável e em tratamento quimio e radioterápico paliativo após a introdução da PEG. Os resultados observados apontaram estabilidade nos valores de índice de massa corporal médio e da avaliação laboratorial, além do aumento da sobrevida de 5,9 meses em 12 pacientes, enquanto outros 3 permaneceram vivos e foram acompanhados pela equipe de nutrição por 6, 14 e 17 meses $^{23}$.

Por não existirem evidências para a decisão de continuar ou suspender a alimentação na fase terminal e por existirem influências culturais envolvendo os alimentos, a decisão de nutrir até a morte deve ser multiprofissional e ter o consentimento do paciente ou de seus familiares, caso não haja condições de decidir por si próprio $^{11}$

\section{CONSIDERAÇÕES FINAIS}

Os consensos e dissensos descritos em literatura científica sobre a indicação e continuidade da TNE nos cuidados paliativos de pacientes com DCNT foram apresentados e ainda repercutem questionamentos sobre os reais benefícios e riscos em promover o suporte nutricional para a população estudada.

No estágio inicial da doença, não foram encontrados dissensos sobre a introdução e continuidade da TNE. Tal situação talvez possa ser explicada pela restauração ou manutenção do estado nutricional contribuir para uma melhor resposta ao tratamento e qualidade de vida do paciente em cuidados paliativos. Entretanto, na fase terminal, é necessário avaliar os reais objetivos da conduta dietoterápica, a qual deverá ser embasada nos princípios de autonomia, beneficência, não maleficência e justiça.

Em síntese, a introdução e a continuidade da TNE nesses pacientes devem ser realizadas de forma individualizada, considerando cada estágio da doença de base, visando a melhoria na qualidade de vida e respeitando os desejos do paciente e de seus familiares. 


\section{REFERÊNCIAS}

1. Malta DC, Moura L, Prado RR, Escalante JC, Schmidt MI, Duncan BB. Mortalidade por doenças crônicas não transmissíveis no Brasil e suas Regiões, 2000 a 2011. Epidemiol Serv Saúde. 2014;23(4):599-8. http://dx.doi.org/10.5123/S1679-49742014000400002

2. Brasil. Ministério da Saúde. Secretaria em Vigilância em Saúde. Departamento de Análise de Situação em Saúde. Plano de ações estratégicas para o enfrentamento das doenças crônicas não transmissíveis (DCNT) no Brasil 2011-2022. Brasília: Ministério da Saúde; 2011.

3. Silva Filho SRB, Lôbo RR, Lima NKC, Ferriolli E, Moriguti JC Cuidados paliativos em enfermaria de clínica médica. Medicina. 2010;43(2):126-33

4. Holmes S. Importance of nutrition in palliative care of patients with chronic disease. Primary Care. 2010;21(6):31-9. DOI: http://dx. doi. org/10.7748/phc2011.07.21.6.31.c8606

5. Conselho Regional de Medicina do Estado de São Paulo (CREMESP). Cuidado Paliativo. Disponível em: http://www. cremesp.org.br. Acesso em 17 abr. 2015.

6. World Health Organization (WHO). Palliative care for older people: better practices. Disponível em: http://www.euro.who.int. Acesso em 17 abr. 2015

7. Druml C, Ballmer PE, Druml W, Oehmichen F, Shenkin A, Singer $P$, et al. ESPEN guideline on ethical aspects of artificial nutrition and hydration. Clinical Nutrition 2016;35(3):545-56 http://dx.doi.org/10.1016/j.clnu.2016.02.006

8. Côrrea PH, Shibuya E. Administração da terapia nutricional em cuidados paliativos. Rev Bras Cancerol. 2007;53(3):317-23.

9. Benarroz MO, Faillace GBD, Barbosa LA. Bioética e nutrição em cuidados paliativos oncológicos em adultos. Cad Saúde Pública. 2009;25(9):1875-82

http://dx.doi.org/10.1590/S0102-311X2009000900002

10. Botelho LLR, Cunha CCA, Macedo M. O método da revisão integrativa nos estudos organizacionais. Gestão Soc. 2011;5(11):121-36.

11. Academia Nacional de Cuidados Paliativos (ANCP). Manual de cuidados paliativos. Rio de Janeiro: Diagraphic; 2012.

12. Brasil. Ministério da Saúde. Instituto Nacional do Câncer (INCA) Consenso Nacional de Nutrição Oncológica. Rio de Janeiro: INCA; 2011.

13. August DA, Huhmann MB, American Society for Parentera and Enteral Nutrition (ASPEN). A.S.P.E.N. Clinical Guidelines: Nutrition Support Therapy During Adult Anticancer Treatment and in Hematopoietic Cell Transplantation. J Parent Enter Nutr. 2009;33(5):472-500

http://dx.doi.org/10.1177/0148607109341804
14. Northamptonshire Healthcare (NHS). Nutritional Screening in Palliative Care. [Internet]. Disponivel em: http://www.nht.nhs.uk/ mediaFiles/downloads/13103460/CLPg010\%20Nutritional\%20 Screening\%20in\%20Palliative\%20Care\%20review\%20May\%2016. pdf. Acesso em: 28 jul. 2016

15. Choudhuri AH. Palliative Care for Patients with Chronic Obstructive Pulmonary Disease: Current Perspectives. Indian J Palliat Care. 2012;18(1):6-11.

http://dx.doi.org/10.4103/0973-1075.97342

16. Planas M, Álvarez J, Gárcia-Peris PA, de la Cuerda C, Lucas P, Castella $\mathrm{M}$, et al. Nutritional support and quality of life in stable chronic obstructive pulmonary disease (COPD) patients. Clin Nutr. 2005;24(3):433-41

http://dx.doi.org/10.1016/j.clnu.2005.01.005

17. Campos LN. Qual a utilização de suplementos orais na prática clínica hospitalar no Brasil e no mundo? [Internet]. 2010. Disponível em:http://humanaalimentar.com.br/custom/308/uploads/pdf/ artigoscientificos/suplementosoraisnobrasil.pdf. Acesso em: 26 ago. 2015

18. Sampson EL, Candy B, Jones L. Enteral tube feeding in older people with advanced dementia: Findings from a Cochrane systematic review. Cochrane Database Syst Rev. 2009;15(2):CD007209. http://dx.doi.org/10.1002/14651858.CD007209.pub2

19. American Geriatrics Society Ethics Committee and Clinical Practice and Models of Care Committee. American Geriatrics Society feeding tubes in advanced dementia position statement. J Am Geriatr Soc. 2014;62(8): 1590-93. http://dx.doi.org/10.1111/jgs.12924

20. Fernadéz BA, Gárcia-Ordoñez MA, Manzanares CM, Huelgas RG Survival of a cohort of elderly patients with advanced dementia: nasogastric tube feeding as a risk factor for mortality. Int J Geriatr Psychiatry. 2005;20(4):363-70. http://dx.doi.org/10.1002/gps.1299

21. Reiriz AB, Motter C, Buffon VR, Scatola RP, Fay AS, Manzini M. Cuidados paliativos - Há benefícios na nutrição do paciente em fase terminal? Rev Soc Bras Clin Med. 2008;6(4):150-5

22. Pasman HRW, Onwuteaka-Philipsen BD, Kriegsman DM, Ooms ME, Ribbe MW, van der Wal G. Discomfort in nursing home patients with severe dementia in whom artificial nutrition and hydration is forgone. Arch Intern Med. 2005;165(15):1729-35. http://dx.doi.org/10.1001/archinte.165.15.1729

23. Grilo A, Santos CA, Fonseca J. Percutaneous endoscopic gastrostomy for nutritional palliation of upper esophageal cancer unsuitable for esophageal stenting. Arq. Gastroenterol. 2012;49(3):227-31. http://dx.doi.org/10.1590/S0004-28032012000300012 